\title{
Case of Corneal Melting with Phthisis Bulbi with Uveal Tissue Prolapse in Left Eye
}

\section{Sonal Muley ${ }^{1}$, Chetan Saoji², Sachin Daigavane ${ }^{3}$, Tejas Sadavarte ${ }^{4}$}

'Senior Resident Dept. of Ophthalmology Datta Meghe Medical College, Shalinitai Meghe Hospital and Research Centre, Hinaganao, Nagpur-441110, MS, India; ${ }^{2}$ Assistant Professor Dept. of Ophthalmology Datta Meghe Medical College, Shalinitai Meghe Hospital and

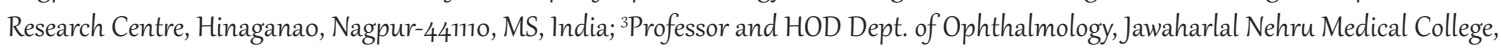
Datta Meghe Institute of Medical Sciences, Wardha-442001, MS, India; ${ }^{4}$ Assistant Professor Dept. of Radiology Datta Meghe Medical College, Shalinitai Meghe Hospital and Research Centre, Hinaganao, Nagpur-441110, MS, India.

\section{ABSTRACT}

Introduction: This is a case of a 26-year-old female who presented to the medicine OPD with fever, chills, periorbital swelling of the left eye associated with discharge. The patient had a history of blood transfusion and severe anaemia (Hb-2.8gm\%). Visual acuity in the right eye was 6/6 with a normal examination. The visual acuity in the left eye was no PL with no retinal glow.

Result: There was the presence of bloody discharge, lid oedema, ecchymosis, chemosis, subconjunctival haemorrhage, hyphema in the anterior chamber, corneal melting and uveal tissue prolapse. MRI Brain and orbit was suggestive of Phthisis Bulbs of the left globe with tuberculoma with ventriculitis with ependymitis. Treatment given was blood and platelet transfusion, iv antibiotics, injection dexamethasone and anti-tubercular drugs. Evisceration of the left eye was done.

Conclusion: Manifestations of tuberculosis in the eye are varied. No part of the eyeball is immune to tuberculosis except the lens. Timely intervention and treatment may help retain the eye. With the advent of keratoprosthesis surgery, early treatment may help save the eye.

Key Words: Ocular Tuberculosis, Epididymitis, Keratoprosthesis, Ecchymosis and hypotony

\section{INTRODUCTION}

\section{Phthisis Bulbs}

It is the degenerative change of globe involving all the tissues. It is due to diminished production of aqueous humour causing reduced intraocular pressure (hypotony) and shrinkage of the globe.

There is typical disorganization of intraocular contents, opaque media, corneal scars, exudate in anterior and posterior chambers, advanced cataracts, destruction of vitreous, scleral thickening cyclitic membrane extends from one ciliary body behind the lens to the other ciliary body, complete detachment of retina; also ossification of bone..$^{1,2}$

\section{Tuberculosis}

M. Tuberculosis, an obligate aerobe, slow-growing, non-motile bacterium, causes tuberculosis. It is transmitted primarily as an airborne aerosol that gains access to susceptible hosts via the lungs and contributes to latent or dormant infection in hosts with normal immune systems. The onset of symptoms may occur after an age-related deterioration of the patient's immune system, illness, or immunosuppressive therapy for other conditions. ${ }^{3,4}$ The organism may invade any tissue or organ within the body in immunocompromised persons, rapid progression of the disease can occur. It sometimes invades the local lymph nodes and spreads to extrapulmonary locations, mostly through hematogenic routes. Usually, infected end-organs have high oxygen stress (apices of the lungs, kidneys, bones, meninges, eye and choroid).

Ocular involvement occurs in patients with tuberculosis in around $1 \%$ to $2 \%$ per cent. This appears to masquerades as other infections and disease processes. The hallmark of extra-pulmonary TB is caseating granuloma and necrosis. Extrapulmonary tuberculosis develops in combination with or in isolation from clinically evident pulmonary $\mathrm{TB}$, with no laboratory evidence of pulmonary infection. ${ }^{5,6}$

\section{Corresponding Author:}

Dr. Chetan Saoji, Assistant Professor Dept. of Ophthalmology, Datta Meghe Medical College, Shalinitai Meghe Hospital and Research Centre, Hinagana0, Nagpur-441110; Email: drsonal.j.muley@gmail.com

ISSN: $2231-2196$ (Print)

Received: 26.06 .2020
ISSN: $0975-5241$ (Online)

Revised: 28.07 .2020
Accepted: 30.08 .2020
Published: 22.09 .2020 


\section{Clinical Presentation}

Systemic tuberculosis patients experience fatigue, weight loss, fever, nausea, night sweats and cough. The disease is typically chronic and insidery. In the eye, it may affect any structure. It may be unilateral or bilateral resulting from the hematogenic spread, direct local extension from the skin, mucous membranes and sinuses or as a reaction to distant infection with hypersensitivity. Posterior uveitis, followed by anterior uveitis, panuveitis, intermediate uveitis tend to be the most common ocular clinical presentations. The sclera is also involved in panophthalmitis, which leads to globe perforation. Other ocular findings are interstitial keratitis, retinitis, scleritis, orbital abscess, optic neuropathy and palsies of cranial nerves, tuberculous retinal vasculitis or Eales disease. ${ }^{6}$

\section{Diagnosis and Treatment}

Diagnosis of ocular tuberculosis is exceedingly difficult because ocular tuberculosis tends to have no findings on chest $\mathrm{x}$-ray or negative tuberculin skin test. To diagnose ocular tuberculosis clinically, subconjunctival tuberculin test or therapeutic isoniazid (INH) test can also be performed. Polymerase chain reaction (PCR) technique has been used to detect mycobacterium in intraocular samples such as aqueous or vitreous humour to confirm the diagnosis of ocular tuberculosis. Retinal vasculitis and tuberculoma are usually responsive to corticosteroid treatment. The mainstay of treatment is antituberculosis agents. ${ }^{7}$

\section{MATERIALS AND METHODS:}

A case of 26 years old female was reported in the medicine OPD with chief complaints of fever with chills and periorbital swelling of the left eye which was associated with redness and discharge of blood. Thus the patient was referred to the ophthalmology department.

- History of headache and rash all over the body.

- History of 5 units of blood transfusion in a private hospital given $2.8 \mathrm{gm} \%$ of haemoglobin according to reports.

\section{No history of redness}

ulcers

\section{trauma to eye}

- General examination was within normal limits. On neurological examination, she was fully alert and oriented and had fluent speech. There were no signs of meningeal irritation. All cranial nerve examination was normal

\section{Ophthalmologic Examination}

Visual acuity and anterior segment evaluation in the right eye was within normal limits
- Visual acuity left eye- NO PL.

- Presence of discharge of blood, lid oedema, ecchymosis, chemosis, subconjunctival haemorrhage, hyphema in the anterior chamber, corneal melting and uveal tissue prolapsed(Fig. 1,2 and 3).

- Fundus examination-

- $\quad$ RE- within normal limits $•$ LE- glow not seen

MRI Brain and orbit was suggestive of Phthisis Bulbi of the left globe with tuberculoma with ventriculitis with ependymitis

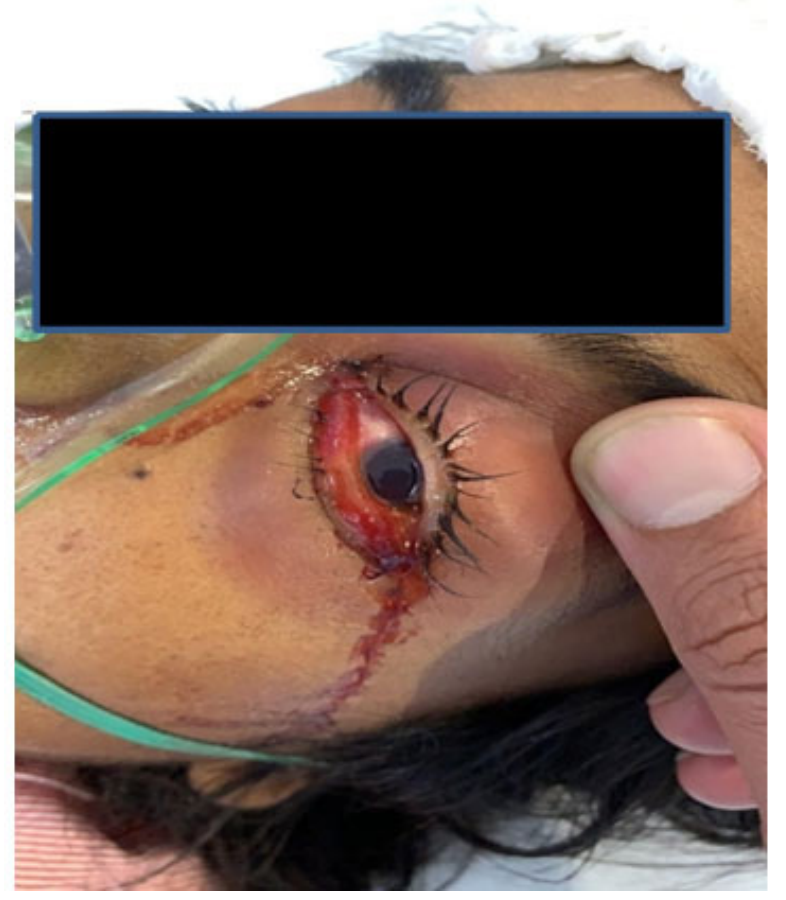

Figure 1: (Day 1) Ecchymosis, chemosis, subconjunctival haemorrhage, discharge.

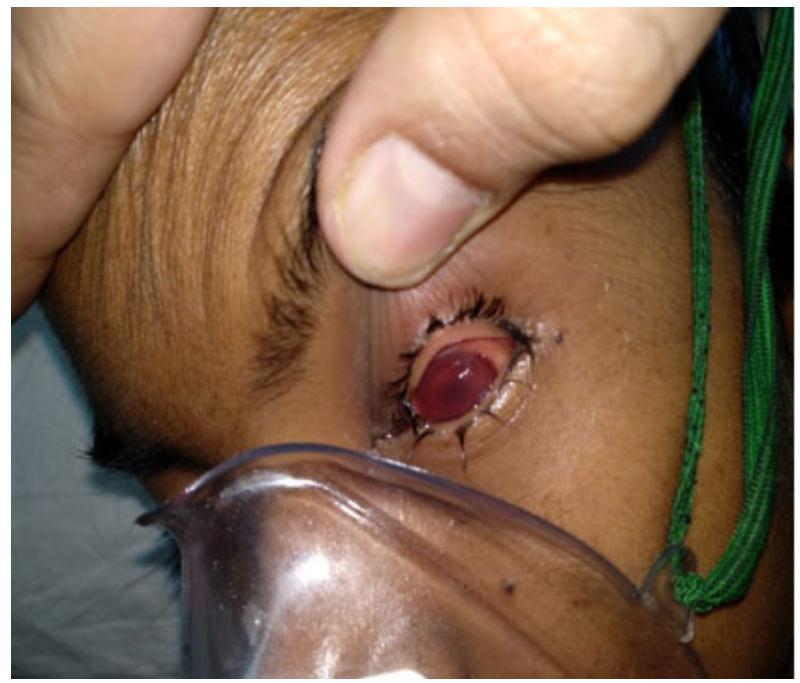

Figure 2: Day two patient developed hyphaema and hypotony. 


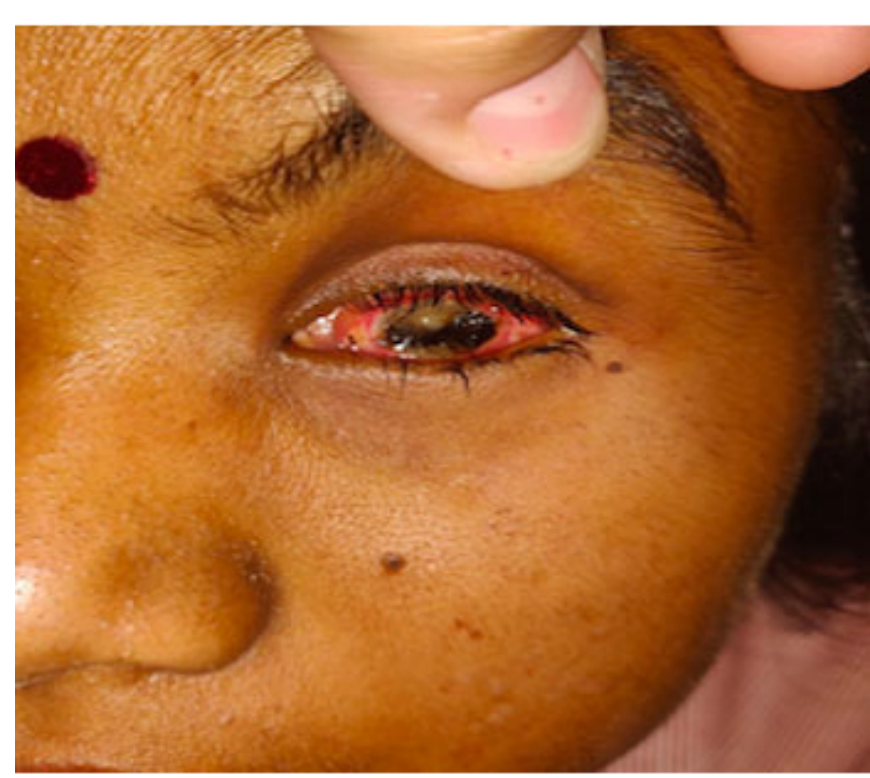

Figure 3: Day four patient developed Phthisis Bulbi with corneal melt and uveal tissue prolapse.

\section{Treatment}

- 2 units of blood transfusion with 13 units of platelet transfusion.

- iv antibiotics were given.

- Injection Dexamethasone $4 \mathrm{mg}$ iv QID x 7 days.

- The patient was started on anti-tubercular drugs. -The evisceration of the left eye was done.

\section{DISCUSSION}

Manifestations of tuberculosis in the eyeball are varied. No part of the eyeball is immune to tuberculosis except the lens.

- The usual parts affected are however conjunctiva, choroid, iris, rarely the optic nerve and the orbital tissue.

- The eye may be affected in three different forms.

- Miliary form-small yellow grey transparent nodules

- Ulcerative form

- Diffuse form. ${ }^{8}$

Tuberculosis can have a varied appearance and timely intervention can go a long way in salvaging the vision and the eye. In a case report by Ashutosh, a 28-year-old woman presented with diminution of vision in both eyes with choroidal tubercles in the RE and a chorioretinal patch in the macula in the left eye. Systemic therapy with steroids and ATT helped gain vision in the RE with the resolution of the choroidal tubercles but $\mathrm{n} 0$ improvement was noted in the left eye. ${ }^{9}$

Ocular tuberculosis can also present as panophthalmitis with rapid progression and phthisis bulbs. In a case report by Savita Agrawal et al, a 26-year-old male with a painful red eye with vision loss in the left eye for the last 1 month. He has also complained about and off about low-grade fever for the last 2 months.
The patient was a known pulmonary TB case and had been on antitubercular medication for the last 2 months. At the time of presentation, the left eye exam revealed no perception of light, circumcorneal congestion with ciliary staphyloma, fixed and dilated pupil, and marked limitation of eye movements. It increased intraocular tension to $29.4 \mathrm{mmHg}$. Other than complicated cataracts, the anterior chamber had exudates, cells $2+$, and flare $2+$. The vision was $6 / 6$ unassisted in the left eye, intraocular stress was within the normal range $(14.6 \mathrm{mmHg})$, and pupillary consensus reaction was absent. ${ }^{10}$

Definitive diagnosis requires that nucleic acid amplification procedures show either AFB in tissue sections or bacterial genome. ${ }^{11}$ A newer test based on anti-cord factor antibody detection by enzyme-linked immunosorbent assay is also used. ${ }^{12}$ Balne et al. Studied multiple factors affecting polymerase chain reaction outcomes in patients with clinically suspected ocular TB. ${ }^{13}$

There are less new TB detection techniques available, such as interferon-gamma release assay, which is focused on the synthesis of gamma interferon by T-cells sensitized to specific MTB antigens and therefore not affected by Calmette - Guérin bacilli and most non-TB bacteria. Such measures include the In-Tube QuantiFERON-TB Gold and ELISpot PLUS. ${ }^{14}$

Techniques for detecting MTB in aqueous and vitreous samples from patients with suspected uveitis TB are also used in the polymerase chain reaction. The most antigenic and abundant cell wall feature of MTB, the detection of antibodies against purified cord factor, may provide clear evidence of the infection. Nonetheless, the sensitivity was reported to be small, as many ocular manifestations may reflect a delayed hypersensitivity reaction rather than a direct mycobacterial infection, resulting in less sensitive analysis of a fluid sample from the eye. ${ }^{14}$

It is recommended that a regular eye test is a must in all susceptible individuals (immunocompromised / identified TB cases). Early antitubercular therapy should be begun on the clinical diagnosis of ocular TB to prevent progression to panophthalmitis and loss of vision.

\section{CONCLUSION}

A significant difference in the clinical appearance and nonspecific ocular TB expression delays the proper diagnosis and thus requires strong clinical suspicion for timely diagnosis. It is recommended that a regular eye test is a must in all susceptible individuals (immunocompromised / identified TB cases). Early antitubercular therapy should be begun on the clinical diagnosis of ocular TB to prevent progression to panophthalmitis and loss of vision. 
Acknowledgement: Authors acknowledge the immense help received from the scholars whose articles are cited and included in references of this manuscript. The authors are also grateful to authors / editors / publishers of all those articles, journals and books from where the literature for this article has been reviewed and discussed.

\section{Conflict of Interest: Nil}

\section{Source of Funding: Nil}

\section{REFERENCES}

1. Pernick N. Phthisis bulbi. PathologyOutlines.com website. https://www.pathologyoutlines.com/topic/eyeglobephthisisbulbi. html. Accessed July 27th, 2020.

2. Pandey S, Satyawali V, Joshi D, Titiyal G. Central Corneal Thickness and Diabetes Mellitus - A Study of Correlation in Terms of Duration and Glycemic Control in North Indian Hilly Population International Journal of Current Research and Review. 2009; 11(14): 01-05.

3. Cladius, S., U. Jadhav, B. Ghewade, S. Ali, and T. Dhamgaye. "Study of Diabetes Mellitus in Association with Tuberculosis." Journal of Datta Meghe Institute of Medical Sciences University 2017;12(2): 143-47.

4. Gupta, V., and Bhake A.. "Clinical and Cytological Features in Diagnosis of Peripheral Tubercular Lymphadenitis - A HospitalBased Study from Central India." Indian Journal of Tuberculosis.2017; 64(4): 309-13.
5. Bagdia M, Bijwe S, Hirani N, Joshi A, Chowdhary A, Agrawal M, Bagdia A. Lab Diagnosis of Extra Pulmonary Tuberculosis: Comparison of Histopathology, Cytology, ZeihlNeelsen stain and Light Emission Diode Microscopy with Culture and Nucleic Acid Amplification Tests International Journal of Current Research and Review. 2017; 10(08): 15-19.

6. Sharma A., Lavaju P. Thapa B. Nepalese journal of ophthalmology: a biannual peer-reviewed academic journal of the Nepal Ophthalmic Society : NEPJOPH .2011;3(5):52-67 .

7. Ishihara M, Ohno S. Nihon Rinsho. 1998;56(12):3157-3161.

8. Shrivastav J.B, Sharma K.D. Tuberculosis of the eyeball, with a case report of tuberculoma of the orbit. Indian J Ophthalmol 1954;2:15-8.

9. Ashutosh. A Rare Presentation of Ocular Tuberculosis: A Case Report. 2017; 5(1): 5556-5563.

10. Agarwal S, Gupta P, Pandey P, Ralli M.Tubercular Panophthalmitis: A case report of a rare entity. Med J DY Patil Univ 2017;10:390-392.

11. Rajpal I, Bhartiya S, Bhargav S. Ocular tuberculosis: Current paradigms in diagnosis and management. DJO 2008;14:23-26.

12. Sharma A, Thapa B, Lavaju P. Ocular tuberculosis: An update. Nepal J Ophthalmol 2011;3:52-67.

13. Balne PK, Modi RR, Choudhury N, Mohan N, Barik MR, Padhi $\mathrm{TR}$, et al. Factors influencing polymerase chain reaction outcomes in patients with clinically suspected ocular tuberculosis. J Ophthalmic Inflamm Infect 2014;4:10.

14. Shakarchi FI. Ocular tuberculosis: Current perspectives. Clin Ophthalmol 2015;9:2223-2227. 